\title{
Heterochronic genes control the stage-specific initiation and expression of the dauer larva developmental program in Caenorhabditis elegans
}

\author{
Zhongchi Liu and Victor Ambros \\ Department of Cellular and Developmental Biology, Harvard University, Cambridge, Massachusetts 02138 USA
}

\begin{abstract}
We report that a stage-specific developmental program, dauer larva formation, is temporally regulated by four heterochronic genes, lin-4, lin-14, lin-28, and lin-29. The effects of mutations in these four genes on dauer larva formation have revealed that they regulate two different processes of dauer larva formation: (1) a decision specifying the larval stage at which dauer larva development initiates, and (2) the specialized differentiation of hypodermal cells during dauer larva morphogenesis. Epistasis analysis has suggested a model in which lin-4 negatively regulates lin-14, and the resulting temporal decrease in lin-14 activity specifies the stage of dauer larva initiation. Our results further suggest that dauer larva morphogenesis by hypodermal cells requires that lin-28 acts to inhibit lin-29 during early larval stages.
\end{abstract}

[Key Words: Heterochronic genes; developmental timing; dauer larva; C. elegans]

Received August 18, 1989; revised version accepted October 10, 1989.

Fundamental to the understanding of animal development is the question of how temporal information is elaborated and how the various cell types use that information for the temporal coordination of diverse developmental events. In Caenorhabditis elegans, genes that encode components of a temporal control system have been identified by mutations that specifically disrupt the normal sequence of postembryonic developmental events (Ambros and Horvitz 1984). This class of genes has been termed 'heterochronic' by analogy with the evolutionary concept of heterochrony, which refers to interspecific differences in the relative timing of developmental events (Gould 1977).

Mutations in the heterochronic genes lin-4, lin-14, lin-28, and lin-29 cause temporal transformations in cell fates, resulting in either 'precocious' development, in which certain cells express fates that normally occur later in development, or 'retarded' development, in which cells reiterate fates normally specific for earlier stages. Thus, these genes control the time during development when cells select specific fates from among a series of developmental alternatives. Because heterochronic genes control choices of cell fates in a temporal context, they are members of a general class of developmental 'switch' genes, which also include genes for pattern formation and sex determination in Drosophila (Lewis 1978; Baker and Ridge 1980; Ingham 1988) and C. elegans (Hodgkin 1980, 1985; Greenwald et al. 1983; Kenyon 1986; Miller et al. 1988).

Analysis of the stage and lineage specificity of the four heterochronic genes has revealed a phenotypic hierarchy (Ambros and Horvitz 1984). lin-4 and lin-14 mutations affect the timing of events in diverse cell lineages, whereas lin-28 and lin-29 mutations affect a restricted subset of the events and the cell lineages affected by lin-4 and lin-14. One particular stage-specific event in the development of the C. elegans lateral hypodermis is the larva-to-adult (L/A) switch, which involves coordinate changes from the larval fate to the adult fate in lateral hypodermal 'seam' cells (Ambros 1989). The seam cells are hypodermal stem cells that divide during larval development and cease division and differentiate at the larva-to-adult molt. The timing of this L/A switch is affected by mutations in all four heterochronic genes. Epistasis analysis of the effects of these four heterochronic genes on the timing of the L/A switch has revealed a functional genetic hierarchy. lin-4 appears to be a negative regulator of $l i n-14$ and lin-28; lin-14 and lin-28 act together to negatively regulate $l i n-29$, a gene required for the execution of the L/A switch (Ambros 1989). These findings have suggested that lin-4 and lin-14 are general temporal control genes responsible for the elaboration of temporal information for diverse cell types, and other genes, such as lin-29, are likely genes responsible for the use of temporal information for tissue-specific and stage-specific choices of cell fates.

Here, we explore whether or not the general temporal control genes, lin-4 and lin-14, affect developmental events distinct from the L/A switch by the same genetic pathway. By comparing the genetic circuitry by which 
different developmental events are temporally regulated, one may address how heterochronic genes interact to coordinate the temporal control of multiple developmental events in diverse cell types and at different developmental stages.

We have chosen to focus on a stage-specific developmental event, dauer larva formation. The dauer larva is a morphologically specialized and developmentally arrested third-stage larva. The choice between developing into a dauer larva or developing continuously through the normal four larval stages is controlled by environmental signals, including high temperature, starvation, and crowding (Cassada and Russell 1975; Golden and Riddle 1984), and is also under rigorous temporal control-wild-type animals can enter the dauer larva stage only at the second larval molt. Animals that develop past the second larval stage lose the ability to become dauer larvae even under stressed culture conditions. The rigid environmental and temporal control of such an alternative developmental program and the convenient selection method for isolating dauer larvae (Cassada and Russell 1975) offer an excellent model system to study the genetic control of a simple developmental switch and to address the question of how the integration of environmental and temporal information is achieved.

A great amount of work has been done on the environmental control of dauer larva formation (Riddle 1988). Two classes of daf (dauer formation) mutations have been identified that disrupt the environmental control of dauer larva formation. One class is dauer-constitutive mutants, which form dauer larvae even in the presence of abundant food (Swanson and Riddle 1981). In contrast, dauer-defective mutants are unable to form dauer larvae even under conditions of starvation, crowding, and high temperature (Riddle 1977; Albert et al. 1981). None of the daf mutations alters the developmental stage at which dauer larva development is initiated; the genes defined by daf mutations are apparently not involved in the temporal regulation of the decision to initiate the dauer larva program.

We examined the effects of mutations in the four heterochronic genes on the temporal control of dauer larva development. Our analysis of the phenotypes of single and multiple heterochronic mutants has revealed that lin-4 and lin-14 regulate the stage at which the dauer larva program initiates. lin-28 and lin-29 do not regulate the stage specificity of dauer larva initiation, but do affect dauer larva morphogenesis. We discuss the significance of these findings to the control of multiple developmental events in different tissues, and the integration of temporal and environmental information for dauer larva initiation.

\section{Results \\ Dauer larva formation in the wild type}

Dauer larva formation in the wild type is strictly stage specific, occurring only at the second larval molt, and is accompanied by complete developmental arrest and dra- matic morphological changes, including radial constriction of the body and the formation of a dauer larva-specific cuticle (Cassada and Russell 1975). The dauer larva cuticle is physically and morphologically specialized and is easily distinguished by characteristic raised longitudinal ridges, or lateral alae (Cassada and Russell 1975; Fig. 1a). Dauer larvae do not feed, and they acquire resistance to harsh conditions such as detergent treatment (Cassada and Russell 1975). After extended periods of developmental arrest, dauer larvae can, on exposure to favorable culture conditions, resume feeding and complete the remainder of larval development. In the experiments described, the acquisition of resistance to treatment with SDS and the presence of morphologically distinct lateral alae are the major criteria used to identify animals expressing the dauer larva developmental program (see Methods).

\section{Mutations in heterochronic genes cause two distinct abnormalities in dauer larva development}

Dauer larvae formed at abnormal larval stages In contrast to wild-type animals, which form dauer larvae solely at the second larval molt, heterochronic mutants can form dauer larvae at the first larval molt (precocious dauer larvae) or at the third larval molt (retarded dauer larvae). For most experiments, we determined the developmental stage of dauer larva formation in heterochronic mutants by examining the extent of gonad development. The timing of the major morphogenetic events in gonad development is not affected in these particular heterochronic mutants (Ambros and Horvitz 1984). Thus, gonad morphology can serve as a reference for developmental stage (Kimble and Hirsh 1979; Ambros and Horvitz 1984). Precocious dauer larvae exhibit a gonad with 8-12 nuclei and an ellipsoid morphology (Fig. 1d-f), typical of animals at the first larval (L1) molt. Dauer larvae formed at the wild-type stage (L2 molt) exhibit a gonad with 20-22 nuclei and an elongated but unreflexed morphology (Fig. 1a-c). Retarded dauer larvae exhibit a gonad with $>100$ nuclei and two arms just starting to reflex (Fig. $1 g-i$ ). To confirm that the gonad morphology is a reliable criterion for staging, we followed the development of representative precocious mutant animals beginning at the time of hatching (see Methods) and observed dauer larva formation at the first molt (data not shown). Aside from differences in gonad morphology and body size, precocious and retarded dauer larvae are similar to wild-type dauer larvae in various other characteristics, including complete developmental arrest, SDS resistance, radial constriction of the body, and the appearance of characteristic lateral alae on the cuticle.

Incomplete dauer larvae A distinctive abnormality found in dauer larvae of certain heterochronic mutants is the failure of individual hypodermal cells or sets of adjacent hypodermal cells to fully exhibit dauer larva morphology. The affected areas of hypodermis lack both the radially constricted morphology and the dauer larva- 


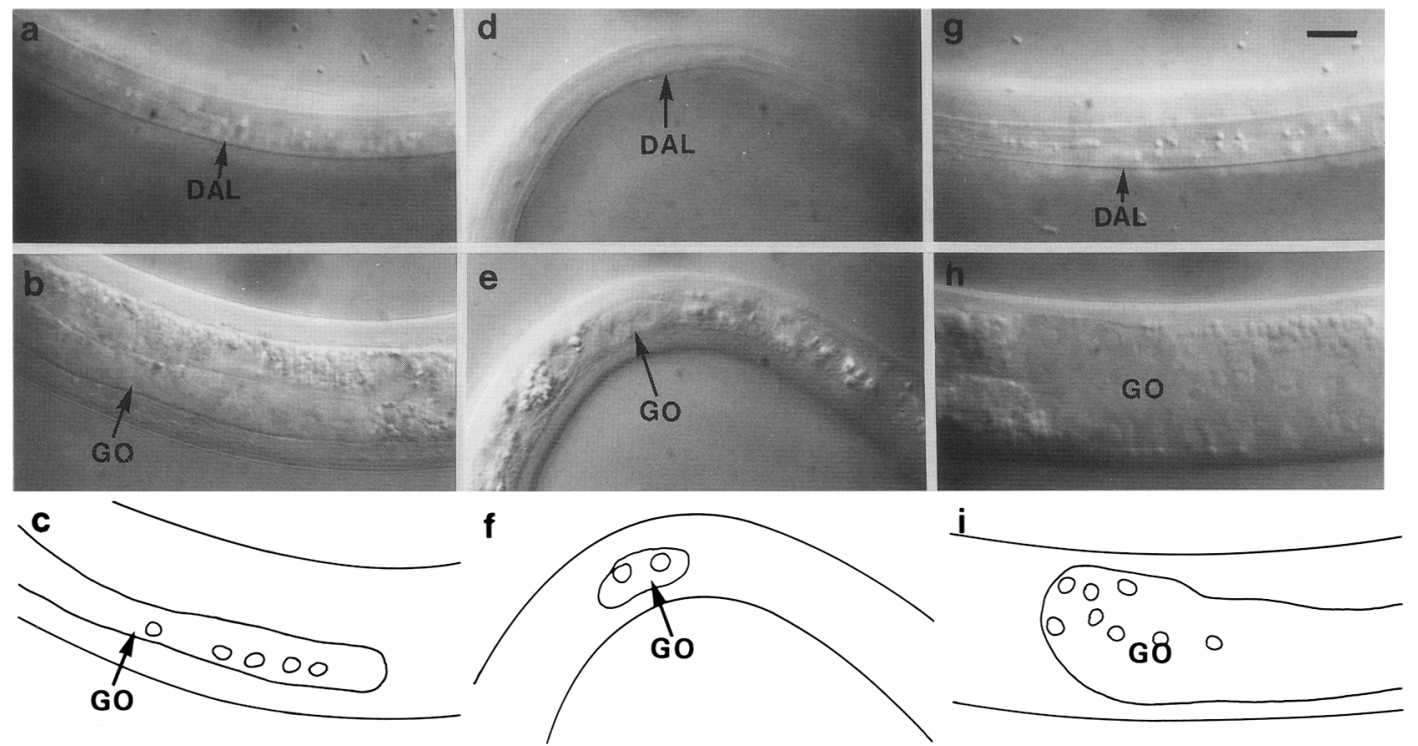

Figure 1. Photomicrographs of living wild-type and heterochronic dauer larvae (Nomarski optics; bar, $20 \mu \mathrm{m}$ ). Each vertical series of photomicrographs is of the same hermaphrodite taken $\sim 5-10 \mathrm{sec}$ apart, but in two focal planes. For each animal, one plane of focus $\mid a$, $d$, and $g$ ) shows the presence of dauer-specific lateral alae (DAL) on the cuticle; another plane $(b, e$, and $h \mid$ shows the gonad $(G O)$ morphology, which serves as a reference for the developmental stages. (c, $f$ and $i)$ Tracings of the corresponding photomicrography, $b$, $e$, and $h$; only representative gonad nuclei are illustrated. $(a-c)$ A wild-type dauer larva that is formed at the L2 molt with a gonad of 20-22 nuclei and two elongating arms (one arm is not completely shown). (d-f) a lin-14(lf) [MT1848 lin-14(n360)] dauer larva that was formed precociously at the $\mathrm{L} 1 \mathrm{molt}$. This animal possesses an ellipsoid gonad containing $8-12$ nuclei (at this focal plane, only two nuclei are visible), a morphology characteristic of $\mathrm{L} 1$ molt animals. $(\mathrm{g}-\mathrm{i})$ a $\operatorname{lin}-14(\mathrm{~g} f /+)$ retarded dauer larva [VT181 lin-14(n536) dpy-6(e14)/szT1]. This animal possesses a highly developed gonad of $>100$ nuclei. Shown here is the growing tip of one of the gonad arms, which is in the process of reflexing. All three dauer larvae were SDS-resistant and were isolated from starved cultures.

specific lateral alae (Fig. 2). These incomplete dauer larvae are developmentally arrested and, in most cases, SDS resistant. In general, the areas of incomplete morphogenesis occur in variable hypodermal regions of the animal, and the size of the incomplete area varies from a single cell to groups of adjacent cells.

\section{lin-14 temporally regulates dauer larva initiation}

Two distinct classes of Iin-14 alleles have been isolated: recessive, loss-of-function (If) mutations and semidominant, gain-of-function $(\mathrm{g} f$ ) mutations (Ambros and Horvitz 1984, 1987). The loss-of-function mutations result in precocious expression of cell lineages in diverse cell types, whereas the gain-of-function mutations result in retarded expression of cell lineages in the same cell types. The genetic and molecular studies of lin-14 have suggested a model (Ambros and Horvitz 1987; Ruvkun and Giusto 1989) wherein lin-14 activity decreases from high to low during development and this reduction leads to the expression of specific cell fates at appropriate stages. lin-14(lf) mutations result in a low level of lin-14 activity throughout development, leading to the precocious expression of cell fates normally specific for later development. lin-14(gf) mutations appear to maintain a high level of lin-14 throughout development, resulting in reiteration of normally early fates.

lin-14 plays a similar role in regulating dauer larva initiation. In high-density and starved cultures, animals homozygous for lin-14(lf) mutations can arrest development as dauer larvae at the first larval molt $\langle\mathrm{L} 1\rangle$, one stage earlier than the wild type (Fig. 1d-f; Table 1A). A single starved culture of lin-14(lf) mutants contains mixtures of dauer larvae formed at the Ll molt and dauer larva formed at the L2 molt. A total of seven lin-14(lf) mutations have been examined: $n 727$ (Table 1A), ma135, n179ts, ma145, n360, n536n838, and n355n679ts (data not shown), and all can cause precocious dauer larva formation. We measured the ratio of $\mathrm{L} 1$ molt dauer larvae to the total dauer larvae and observed that this ratio varies from culture to culture. For example, in six independent cultures of $\operatorname{lin}-14(n 727)$, the

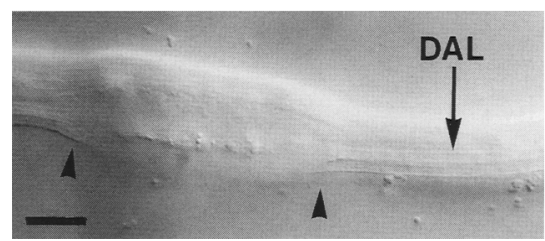

Figure 2. Photomicrograph of an incomplete dauer larva (Nomarski optics; bar, $10 \mu \mathrm{m})$. Shown is an MT1524 lin-28(n719) dauer larva formed at the L2 molt and isolated from a starved culture by SDS selection (see Methods). The area between the two arrowheads lacks both dauer-specific lateral alae (DAL) and the radial constriction. lin-14(lf), lin-14(gf/ + l, and lin-4(e912); lin-14 (n179ts) mutants also form incomplete dauer larvae with a morphology similar to the one shown here. 
Table 1. Stages of dauer larva initiation in heterochronic mutants

\begin{tabular}{|c|c|c|c|c|c|}
\hline \multirow[b]{2}{*}{ Strains } & \multirow[b]{2}{*}{ Genotype $^{a}$} & \multicolumn{3}{|c|}{$\operatorname{Molt}(\%)^{\mathrm{b}}$} & \multirow{2}{*}{$\underset{(n)}{\text { Comments }^{\mathrm{c}}}$} \\
\hline & & $\mathrm{L} 1$ & $\mathrm{~L} 2$ & L3 & \\
\hline N2 & wild type & 0 & 100 & 0 & $>100$ \\
\hline \multicolumn{6}{|l|}{ A. } \\
\hline MT1851 & $\operatorname{lin}-14(1 f)$ & $3-85$ & $15-97$ & 0 & $>100$ \\
\hline MT1397 & $\operatorname{lin}-14\left(\mathrm{n} 179\right.$ ts $\left.15^{\circ} \mathrm{C}\right)$ & 0 & 100 & 0 & 110 \\
\hline MT1388 & $\operatorname{lin}-14\left(n 355 \mathrm{n} 679 \mathrm{ts} 15^{\circ} \mathrm{C}\right)$ & 0 & 97.5 & 2.5 & 165 \\
\hline VT181 & $\operatorname{lin}-14(g f /+)$ & 0 & $92-88$ & $8-12$ & 275 \\
\hline MT1149 & $\operatorname{lin}-14(g f)$ & 0 & 0 & 0 & defective \\
\hline \multicolumn{6}{|l|}{ B. } \\
\hline СВ912 & $\operatorname{lin}-4(l f)$ & 0 & 0 & 0 & defective \\
\hline MT1999 & $\operatorname{lin}-4(l f) ; \operatorname{lin}-14(l f)$ & 98 & 2 & 0 & 65 \\
\hline VT392 & lin-4(lf); Iin-14(n179ts $\left.15^{\circ} \mathrm{C}\right)$ & 0 & 89 & 11 & 9 \\
\hline \multicolumn{6}{|c|}{ (212 } \\
\hline MT1524 & $\operatorname{lin}-28(l f)$ & 0 & 100 & 0 & $>100$ \\
\hline MT1537 & $\operatorname{lin}-28(l f) ; \operatorname{lin}-14(g f)$ & 0 & 0 & 0 & defective \\
\hline MT1538 & $\operatorname{lin}-28(l f) ; \operatorname{lin}-4(l f)$ & 0 & 0 & 0 & defective \\
\hline VT116 & $\operatorname{lin}-28(1 f) ; \operatorname{lin}-14(1 f)$ & + & + & - & $>50$ \\
\hline \multicolumn{6}{|c|}{ (1) } \\
\hline МT333 & $\operatorname{lin}-29(l f)$ & 0 & 100 & 0 & $>100$ \\
\hline VT394 & $\operatorname{lin}-29(1 f)_{;} ; \operatorname{lin}-28(l f)$ & 0 & 100 & 0 & $>100$ \\
\hline VT390 & lin-29(lf);lin-14(n355n679ts $\left.15^{\circ} \mathrm{C}\right)$ & - & + & + & $>50$ \\
\hline VT395 & $\operatorname{lin}-29(l f) ; \operatorname{lin}-14(g f)$ & 0 & 0 & 0 & defective \\
\hline MT1836 & lin-29(lf);lin-4(lf) & 0 & 0 & 0 & defective \\
\hline VT391 & lin-29(lf);lin-14(lf) & $50-52$ & $48-50$ & 0 & 41 \\
\hline
\end{tabular}

Percentage of dauer larvae formed at the indicated molt in heterochronic mutants. This percentage can vary, depending on the population age at the time of starvation (see text); these numbers are derived from representative cultures of each strain. In cases where more than one culture was examined, ranges of the percentages are shown.

${ }^{a}$ Full genotypes are listed in Methods. At the permissive temperature $\left(15^{\circ} \mathrm{C}\right)$, lin-14(n179ts) animals are wild type. lin-14(n355n679ts) animals exhibit weak retarded defects at $15^{\circ} \mathrm{C}$ (see Methods).

$\mathrm{b} / 0 \%)$ The consistent absence of dauer larva formed at the indicated molt. For strains where dauer larvae were observed at the indicated molt but were not quantified, the presence $(+)$ or the absence $1-1$ of dauer larva is indicated.

$c(n)$ Total number of dauer larvae examined; some numbers are estimations, as depicted by $>$. (defective) Absence of dauer larva formation at any stages under any conditions (also see Methods).

proportion of dauer larvae that were precocious ranged from $3 \%$ to $85 \%$ (Table 1 ). The precocious dauer larva formation in lin-14(lf) mutants indicates that these $1 f$ mutations disrupt a negative regulation of dauer larva formation by the lin-14 gene product at the L1 molt. This loss of negative control enables lin-14(lf) mutants to form dauer larvae at either the L1 or L2 molt. It is likely that the observed ratio of L1 to L2 molt dauer larvae reflects the relative proportion of larvae of different ages in the population at the time of encountering harsh culture conditions. We have tested a synchronized population of lin-14(n727);daf-7(e1372ts) double mutant for dauer larva formation at nonpermissive temperature starting at hatching and observed dauer larva formation at both the L1 and L2 molts (data not shown). This occurrence of dauer larva formation at both the L1 and L2 molts may reflect incomplete penetrance of the dauer-constitutive mutation daf-7(e1372ts) and/or lin-14(n727).

The effect of lin-14(gf) mutations on the timing of dauer larva formation is opposite to the effect of lin-14(If) mutations. The lin-14(gf) mutations delay or, in some cases, inhibit dauer larva formation (Table 1A). Animals heterozygous for a semidominant, gain-of-func- tion mutation $\operatorname{lin}-14(n 536 /+)$ or homozygous for a weak semidominant, gain-of-function mutation (n355n679ts at $15^{\circ} \mathrm{C}$; see Methods) can form dauer larvae at the $\mathrm{L} 3$ molt, one stage later than the wild type (Fig. $1 g-i$; Table 1A). Animals homozygous for semidominant, gain-offunction lin-14 alleles (n536/n536 and n355/n355) do not form dauer larvae at any stage (Table $1 A_{\text {; }}$ see Methods). We interpret this dauer-defective phenotype of homozygous $\operatorname{lin}-14(g f / g f)$ strains as a more severe expression of the retarded dauer larva formation defect expressed by heterozygous $g f /+$ animals. This is presumably caused by a higher increase in lin-14 activity in lin-14(gf/gf) animals than the lin-14(gf/+) animals and is consistent with the fact that $1 \mathrm{in}-14(\mathrm{gf} / \mathrm{gf})$ animals exhibit a more severe retarded defect in other aspects of development than the corresponding heterozygotes (Ambros and Horvitz 1984, 1987). The fact that lin-14(gf) mutations delay or inhibit dauer larva formation indicates that lin-14 must be appropriately reduced to allow dauer larva formation at the L2 molt in wild type. This is consistent with the previously proposed model that a temporal gradient in the level of lin-14 activity specifies the fates expressed by cells at several stages of development (Ambros and Horvitz 1987). 
lin-4 controls dauer initiation by negatively regulating lin-14

The one mutant allele of 1 in $-4, e 912$, is recessive and probably causes at least partial loss of lin-4 gene activity (Chalfie et al. 1981). The phenotype of lin-4(e912) is similar to that of $\operatorname{lin}-14(\mathrm{~g} / \mathrm{gf})$ mutants, causing retarded defects in diverse cell lineages (Chalfie et al. 1981; Ambros and Horvitz 1984) and a complete inability to form dauer larvae (Table 1B). Previous genetic experiments (Ambros 1989) have suggested that $l i n-4$ is a negative regulator of lin-14 in controlling the timing of the L/A switch. Our results suggest that lin-4 plays a similar role in the regulation of dauer initiation: each of the four lin-14(lf) mutations tested here $\left(\mathrm{ma} 135, \mathrm{n} 179 \mathrm{ts}, \mathrm{n} 355 \mathrm{n} 679 \mathrm{ts}\right.$ at $25^{\circ} \mathrm{C}$, and $n 727)$ suppresses the dauer-defective phenotype of lin-4(e912), and leads to the formation of both L1 and L2 molt dauer larvae in the corresponding lin-4(e912); lin14(lf) double mutants (Table 1B). This epistasis of lin-14(1f) alleles over lin-4(e912) indicates that the effect of lin-4 on dauer initiation is mediated through lin-14. Although lin-4(e912) animals are unable to form dauer larvae at any stage, by the use of a partial suppressor of lin-4(e912), we found that lin-4(e912) can cause retarded dauer larva formation. lin-14(n179ts) is a weak hypomorphic allele of lin-14 at the permissive temperature $\left(15^{\circ} \mathrm{C}\right)$ and partially suppresses many of the cell lineage defects of lin-4(e912) (Ambros and Horvitz 1984). Although lin-14(n179ts), itself, has no effect on dauer larva formation at $15^{\circ} \mathrm{C}$ (Table 1A), the lin-4(e912);in14(n179ts) double mutant animals form dauer larvae at both $\mathrm{L} 2$ and L3 molts at $15^{\circ} \mathrm{C}$ (Table 1B). Apparently, the lin-4(e912) mutation causes an elevation of the weakened lin-14 activity and, hence, results in retarded dauer larva formation. The dauer-defective phenotype of lin-4(e912) and the retarded dauer larva defect of lin-4(e912); 1 in-14(n179ts) are likely a result of inappropriate elevation of lin-14 activity and may be analogous to the defect seen in 1 in $-14(g f / g f)$ mutants. Assuming that lin-4(e912) causes loss of lin-4 activity, these observations suggest that the wild-type $\operatorname{lin}-4$ gene product controls the timing of dauer initiation by negatively regulating $\operatorname{lin}-14$.

Initiation of dauer larva development is regulated independent of lin-28 and lin-29

lin-28 loss-of-function mutations cause precocious lateral hypodermal cell lineages and precocious L/A switching; lin-29 loss-of-function mutations cause retarded lateral hypodermal cell lineages and block the
L/A switching (Ambros and Horvitz 1984; Ambros 1989). No gain-of-function mutations exist for these two genes. In contrast to lin-4 and lin-14 mutations, neither lin-28(lf) nor lin-29(lf) mutations alter the stage-specificity of dauer larva initiation (Table 1C,D). Furthermore, lin-28(lf);lin-29(lf) double mutant animals are wild type for dauer larva initiation (Table 1D). Thus, neither lin-28 nor lin-29 is required for the temporal control of dauer larva initiation. Consistent with this idea are the observations (Table 1C,D) that lin-28 and lin-29 mutations do not block the dauer-defective phenotype or the retarded dauer larva formation of $l i n-4(e 912)$ and lin-14(gf/gf) mutants and do not prevent the precocious dauer larva formation of lin-14(lf) mutants. These results indicate that $l i n-4$ and $l i n-14$ regulate dauer larva initiation independent of lin-28 and lin-29 (Fig. 3).

\section{A branched regulatory pathway regulates dauer larva initiation and the $L / A$ switch}

The findings above indicate that the pathway for the temporal regulation of dauer larva initiation branches from the pathway for regulating the L/A switch: lin-4 and $\operatorname{lin}-14$ regulate the stage of dauer larva initiation, and $\operatorname{lin}-4$, lin-14, lin-28, and $\operatorname{lin}-29$ regulate the hypodermal-specific L/A switch (Fig. 3). Thus, although the two events occur at separate stages, dauer larva initiation at the L2 molt and the L/A switch at the L4 molt, they are controlled by a common temporal signal involving the action of $l i n-4$ and $\operatorname{lin}-14$. However, the genetic mechanisms for executing these two events in response to $\operatorname{lin}-4$ and $\operatorname{lin}-14$ seem to be separate. This branched pathway allows for the independent temporal expression of these two events. In fact, certain double mutant combinations can cause opposite heterochronic defects in the two events of the same animal. Specifically, lin-29(lf);lin-14(lf) double mutants exhibit retarded L/A switching and precocious dauer larva initiation; lin-28(lf/lf);lin-4(lf) animals express the L/A switch precociously but never form dauer larva, a retarded defect in dauer larva initiation (Table 2). The precocious expression of the L/A switch and retarded dauer initiation (or vice versa) in the same animal suggests that the L/A switch and dauer larva initiation are executed independently and are not causally related.

\section{lin-28 and lin-29 affect morphogenesis of the dauer larva hypodermis}

Although lin-28 loss-of-function mutations do not alter the stage specificity of dauer larva formation, they can

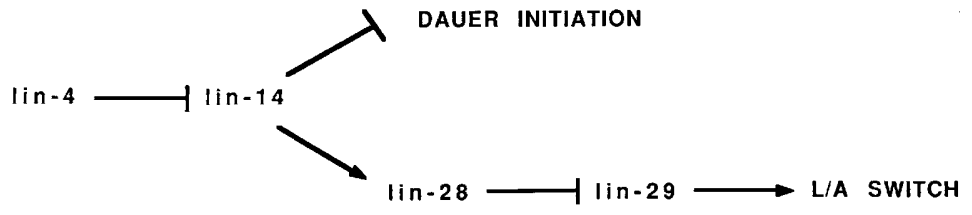
trol the timing of the L/A switch (Ambros 1989), do not participate in the regulation of dauer larva initiation. Arrows indicate positive regulatory interaction; bars, negative regulatory interaction.
Figure 3. A model for the functional relationship between heterochronic genes in regulating the stage specificity of dauer larva initiation and the L/A switch, based on the data in Table 1 and Ambros (1989). lin-4 is a negative regulator of $l i n-14$ which, in turn, negatively regulates dauer initiation. lin-28 and lin-29, which con- 
Table 2. Comparisons of the heterochronic defects in dauer larva initiation and the $L / A$ switch in heterochronic mutants

\begin{tabular}{|c|c|c|}
\hline Genotype $^{a}$ & $\begin{array}{l}\text { Dauer } \\
\text { initiation }\end{array}$ & $\begin{array}{l}\text { L/A } \\
\text { switch b,c }\end{array}$ \\
\hline \multicolumn{3}{|l|}{ Single mutants } \\
\hline $\operatorname{lin}-4(l f)$ & $\begin{array}{l}\text { retarded } \\
\quad \text { (defective) }\end{array}$ & retarded \\
\hline $\operatorname{lin}-14(g f)$ & $\begin{array}{l}\text { retarded } \\
\quad \text { (defective) }\end{array}$ & retarded \\
\hline $\operatorname{lin}-14(1 f)$ & precocious & precocious \\
\hline $\operatorname{lin}-28(1 f)$ & wild type & precocious \\
\hline $\operatorname{lin}-29(l f)$ & wild type & retarded \\
\hline \multicolumn{3}{|l|}{ Double mutants } \\
\hline $\operatorname{lin}-28(l f) ; \operatorname{lin}-4(1 f)$ & $\begin{array}{l}\text { retarded } \\
\text { (defective) }\end{array}$ & precocious \\
\hline $\operatorname{lin}-28(l f) ; \operatorname{lin}-14(g f)$ & $\begin{array}{l}\text { retarded } \\
\text { (defective) }\end{array}$ & precocious \\
\hline $\operatorname{lin}-29(1 f) ; \operatorname{lin}-14(1 f)$ & precocious & retarded \\
\hline
\end{tabular}

Certain double combinations of heterochronic mutations cause opposite heterochronic defects in dauer larva initiation and the L/A switch of the same mutant animal.

alin-4(lf) $=e 912 ; \operatorname{lin}-14$ (lf) $=\operatorname{ma135} ; \operatorname{lin}-14(\mathrm{gf})=n 536$; lin-28(lf) $=n 719 ;$ and lin-29(lf) $=n 1440$.

bPrecocious and retarded refer to the expression of the corresponding event at earlier or later times than the wild type. Defective refers to the absence of dauer larva formation at any stages under any conditions and is considered an extreme retarded defect in dauer larva initiation (see text).

cThe L/A switch phenotype was documented in Ambros and Horvitz (1984).

lead to incomplete dauer larva morphology: animals contain local areas of hypodermis that lack dauer larvaspecific alae and the radial constriction of the body (Fig. 2; Table 3A). The ratio of incomplete lin-28(lf) dauer larvae to the total number of dauer larvae is characteristic of each lin-28(lf) allele and is not appreciably affected by differences in culture conditions. Specifically, six independent starved lin-28(n719) cultures gave ratios ranging from $73 \%$ to $95 \%$ (Table 3); whereas lin-28(n947) cultures consistently gave $\sim 15 \%$ incomplete dauer larvae (data not shown).

The incomplete dauer larva morphogenesis caused by lin-28(lf) mutations appears to be mediated by lin-29. lin-29(lf) mutations suppress the incomplete dauer larva formation caused by lin-28(lf) mutations [lin-29(If) mutations alone do not cause incomplete dauer larva formation; Table 3A]. For example, $73-95 \%$ of lin-28(n719) dauer larvae exhibit incomplete morphology, whereas $0-3 \%$ of lin-28(n719);lin-29(n1440) dauer larvae exhibit incomplete morphology (Table 3A, D). Thus, lin-28(lf) mutations seem to cause the inappropriate expression of lin-29 at earlier larval stages than normal, and this inappropriate lin-29 expression inhibits dauer larva morphogenesis. These observations indicate that one function of the wild-type lin-28 gene product is to prevent lin-29 expression during early larval stages and thus permit complete dauer larva morphogenesis. This is consistent with the previously reported role of lin-28 as a negative regulator of lin-29 during early larval stages to inhibit the execution of the L/A switch until the L4 stage (Ambros 1989). Thus, lin-29 not only acts as an activator of the L4-specific L/A switch but also as an inhibitor of events that occur at earlier larval stages, particularly dauer larva morphogenesis.

\section{lin-4 and lin-14 mutations also cause incomplete dauer larva formation}

Some of the lin-14(lf) dauer larvae formed at L1 and L2 molts, and some of the lin-14/gf/ + ) dauer larvae formed at L2 and L3 molts can exhibit incomplete dauer morphology (Table 3A). Although lin-4(e912) animals do not form dauer larvae, a lin-4(e912);in-14(n179ts) double mutant strain does form dauer larvae at the permissive temperature $\left(15^{\circ} \mathrm{C}\right)$, and portions of these dauer larvae also exhibit incomplete morphology (Table 3B). Because the $n 179$ ts mutation alone does not cause incomplete dauer larvae at the permissive temperature (Table 3A), the incomplete dauer larva morphogenesis of the lin-4(e912);lin-14(n179ts) double mutant suggests that lin-4(e912) affects dauer larva morphogenesis, presumably by elevating lin-14.

Although the incomplete dauer larvae caused by lin-14(lf), lin-14(gf), and lin-4(e912) mutations appear superficially similar in morphology to those caused by lin-28(If) mutations, their incomplete morphogenesis is

Table 3. Dauer larva morphogenesis in heterochronic mutants

\begin{tabular}{|c|c|c|}
\hline Genotype & $\begin{array}{l}\text { Percent incor } \\
\text { dauer larvae }\end{array}$ & $\begin{array}{l}\text { aplete } \\
\qquad(n)^{\mathrm{a}}\end{array}$ \\
\hline \multicolumn{3}{|l|}{ A } \\
\hline $\begin{array}{l}\text { wild type } \\
\text { lin-4(e912) }\end{array}$ & $\begin{array}{c}0 \\
\text { defective }\end{array}$ & $(>100)$ \\
\hline lin-14(n179ts $\left.15^{\circ} \mathrm{C}\right)$ & 0 & $(>100)$ \\
\hline $\operatorname{lin}-14(m a 135)$ & 100 & (23) \\
\hline $\operatorname{lin}-14\left(n 355 n 679\right.$ ts $\left.15^{\circ} \mathrm{C}\right)$ & 65 & (39) \\
\hline $\operatorname{lin}-28(n 719)$ & $73-95$ & $(>100)^{*}$ \\
\hline $\operatorname{lin}-29(n 1440)$ & 0 & $(>100)$ \\
\hline \multicolumn{3}{|l|}{ B } \\
\hline $\operatorname{lin}-4(e 912) ; \operatorname{lin}-14\left(n 179 t s 15^{\circ} \mathrm{C}\right)$ & 66 & (9) \\
\hline \multicolumn{3}{|l|}{$\mathrm{C}$} \\
\hline $\begin{array}{l}\operatorname{lin}-29(n 1440) ; \operatorname{lin}-14(\operatorname{ma} 135) \\
\operatorname{lin}-29(n 1440)\end{array}$ & 83 & $(23)$ \\
\hline $\operatorname{lin}-14\left(n 355 n 679\right.$ ts $\left.15^{\circ} \mathrm{C}\right)$ & 42 & $(8)$ \\
\hline \multicolumn{3}{|l|}{$\mathrm{D}$} \\
\hline lin-29(n1440);lin-28(n719) & $0-3$ & $(>100)^{*}$ \\
\hline
\end{tabular}

The percentage of dauer larvae with incomplete morphogenesis was quantitated by measuring the ratio of SDS-resistant, incomplete dauer larvae to the total number of SDS-resistant dauer larvae (see Methods).

${ }^{a}(n)$ Total number of dauer larvae examined; some numbers are estimations, as depicted by $>.\left({ }^{*}\right)$ Multiple (three to six) cultures were independently selected with $1 \%$ SDS, and the ranges of percentage incomplete dauer larvae are shown. Other percentages were derived from pooled cultures (usually three cultures) that were SDS-selected to give one number. Dauer larvae with very small patches of incomplete morphogenesis could occasionally have been missed using the dissecting microscope; hence, actual percentages may be slightly higher. 
not appreciably suppressed by lin-29(If) (Table 3C). Thus, the effect of lin-14 and lin-4 mutations on dauer larva morphogenesis is attributable to a mechanism different from that of lin-28 and lin-29. Perhaps the lin-4 and lin-14 defect results from partial initiation of the dauer larva program in the animal, whereas the incomplete dauer larva morphogenesis of lin-28 mutants results from the inability of individual hypodermal cells to execute some step(s) in the dauer larva morphogenesis after initiation.

\section{Discussion}

lin-4 and lin-14 regulate the stage specificity of dauer larva initiation

Our primary finding is that the level of lin-14 controls the developmental stage at which dauer larva formation occurs in C. elegans. The effects of lin-14(If) and lin-14(gf) mutations on the stage specificity of dauer larva formation indicate that lin-14 acts as a negative regulator of dauer larva initiation. We propose that during wild-type development, lin-14 level decreases from high during the L1 stage to appropriately low (or absent) at the L2 stage to allow the initiation of dauer larva development. This temporal decrease in lin-14 level is caused by the negative regulation of lin-14 by lin-4. This proposal is consistent with the roles of lin-4 and lin-14 in the temporal regulation of other developmental events, including the expression of stage-specific cell lineage patterns and the L/A switch (Ambros and Horvitz 1984, 1987; Ambros 1989; Ruvkun and Giusto 1989).

The role of lin-14 outlined above as a negative regulator of dauer larva formation in the $\mathrm{L} 1$ accounts for how lin-14 prevents dauer larva formation at the $\mathrm{L} 1 \mathrm{molt}$. A decrease in lin-14 level allows dauer larva formation at the L2 molt, but how does lin-14 specify dauer larva formation only at the L2 molt and not at the L3 molt in some animals? To account more fully for this stage specificity of dauer larva initiation, we further propose that dauer larva initiation is prevented at the L3 molt and later stages by a separate inhibitory activity. Because lin-14/gf/ + ) mutations can allow dauer larva initiation at the L3 molt, the proposed 'L3 inhibitor' would be regulated by lin-14, at least partly.

An alternative model would propose that lin-14 level has to be within a critical range (not too low or too high) to initiate the dauer larva program. Our observations are not consistent with this latter model. If this model were true, a complete loss or a severe reduction of lin-14 activity would abolish dauer larva initiation altogether. However, we have not observed such an effect. We tested seven different lin-14(If) alleles representing four classes of lin-14 loss-of-function mutations, based on their effects on two mutationally separable lin-14 functions, lin-14a and lin-14b (Ambros and Horvitz 1987): $\left(a^{-} b^{-}\right)$, n179ts, ma135, ma145; $\left(a^{+} b^{-}\right)$, n360, n727; $\left(a^{-} b^{+}\right), n 536 n 838$; and $\left(a^{-} b^{++}\right), n 355 n 679 t s$ at $25^{\circ} \mathrm{C}$. We found that all of these mutants were capable of forming dauer larvae at both the L1 and L2 molts. Among these mutations, ma135 appears to be a null or near-null allele based on previous genetic analysis of the L/A switch (Ambros 1989). Thus, lin-14 activity plays only a negative role, to inhibit dauer initiation at the Ll molt and plays no positive role in dauer larva initiation. Furthermore, the fact that both lin-14 $a^{-} b^{+}$, and lin-14 $a^{+} b^{-}$ mutants can form precocious dauer larvae indicates that neither lin-14a nor lin-14b activity alone can prevent dauer larva formation at the $\mathrm{Ll}$ molt.

\section{Evolutionary heterochrony in arrested larval forms}

Because the temporal specificity of a complicated developmental program such as dauer larva formation can be altered by a simple mutation in genes such as lin-14 and lin-4, it is possible that analogous changes in the stage specificity of developmental events among different nematode species may result from simple mutations. Such evolutionary variation in the stage specificity of developmentally arrested infective forms (analogous to C. elegans dauer larvae) have been observed in certain parasitic nematodes (Osche 1963; Evans and Perry 1976; Taylor and Sasser 1978): The developmentally arrested infective form of Meloidogyne incognita occurs at the first molt-one stage earlier than the C. elegans dauer larva; the Ditylenchus vidipsaci infective form occurs at the third molt-one stage later than the C. elegans dauer larva. All of these parasitic nematodes have a total of four larval stages. Our observation of heterochronic dauer larva formation in lin-4 and lin-14 mutants and its interesting analogy to the heterochronic variations among different nematode species support the proposal that heterochrony is a significant mechanism for evolutionary change in life history (Gould 1977).

\section{Dauer initiation and differentiation are controlled by separate genetic pathways}

Our studies of the two kinds of abnormal dauer larvae, dauer larvae formed at abnormal stages and dauer larvae with incomplete morphogenesis, have illuminated two different processes of dauer larva formation: (1) dauer larva initiation and (2) the differentiation of hypodermal cells during dauer larva morphogenesis (Albert and Riddle 1988; Riddle 1988). Mutations in lin-4 and lin-14 alter the stage at which dauer larva development is initiated. We interpreted this effect as reflecting a role of lin-4 and lin-14 in a global decision to initiate a dauer larva developmental program. Mutations in lin-28 and lin-29 do not affect the stage specificity of dauer initiation but do affect dauer larva morphogenesis. Thus, the incomplete dauer larva formation caused by lin-28(lf) mutations reflects a process distinct from the decision to initiate dauer larva development. Because radial constriction and the formation of dauer larva-specific lateral alae are expressed by lateral hypodermal seam cells (Singh and Sulston 1978), the incomplete dauer larva morphology of lin-28(lf) mutants may reflect a defect in the seam cells in particular. The observation that the incomplete areas do contain seam cell nuclei suggests 
that those areas do not suffer from cell death or other dramatic cell lineage alterations. Thus, the failure of those seam cells to fully exhibit dauer larva morphology likely results from an inappropriate temporal identity in those cells as a result of an altered lin-28 activity and, consequently, an inappropriate expression of lin-29. The occurrence of a mixture of affected and unaffected cells in the same animal would appear to be a consequence of the variable expressivity of heterochronic defects previously observed for defects in cell lineages and the L/A switch (Ambros and Horvitz 1984, 1987).

We observed that lin-4 and lin-14 mutations also cause the formation of dauer larvae with incomplete morphology. However, unlike the incomplete dauer larvae of lin-28(lf), the incomplete morphology of lin-4(e912), lin-14(lf), and lin-14(gf) dauer larvae is not suppressed by lin-29 mutations. A simple interpretation of these findings is that lin-4 and lin-14 affect dauer larva morphogenesis by causing cells to receive a weak and/or variable dauer initiation signal, resulting in the occurrence, in the same animal, of cells that initiate dauer larva program and cells that do not. In contrast, lin-28(lf) mutations cause cells that have initiated dauer larva development to be blocked at a subsequent step of morphological differentiation, e.g., differentiation of a radially constricted cuticle.

The fact that lin-28 and lin-29 affect dauer larva morphogenesis and not the stage specificity of dauer larva initiation suggests a separation or branching of genetic pathways for the control of dauer larva initiation and differentiation. Similarly, the regulatory pathway for dauer larva initiation is also partly separated from that for the L/A switch (Fig. 3). These genetic separations indicate that dauer larva initiation may occur at a different time in development and/or in different tissues from dauer larva differentiation and the L/A switch. The fact that lin-28 and lin-29 mutations affect both the hypodermal-specific L/A switch (Ambros and Horvitz 1984) and ability of the hypodermal seam cells for dauer larva differentiation suggest that lin-28 and lin-29 may encode regulators specific to hypodermal cells.

The initiation of dauer larva formation in response to environmental signals (food, pheromone, temperature) seems to be mediated by specific neurons (Lewis and
Hodgkin 1977; Albert et al. 1981; C. Bergman and R. Horvitz, pers. comm.). Thus, lin-4 and lin-14 may regulate the stage of dauer larva formation by controlling a signal (or the response to a signal) originating from particular neurons. This signal would trigger other cells in the animal to commit to dauer larva development. This hypothesis is consistent with the observation of neuronal staining with antibody to a lin-14 protein in late L1 larvae (Ruvkun and Giusto 1989). Dauer larva morphogenesis is likely to reflect a process occurring within individual cells in response to the global signal discussed above. Accordingly, dauer larva morphogenesis may consist of many steps (Riddle 1988), including the reception of the signal by individual cells and the expression of genes required for the specialized differentiation of the dauer larva. Figure 4 incorporates these ideas into a model for the temporal regulation of distinct aspects of dauer larva development by lin-4, lin-14, lin-28, and lin-29. In this model, lin-4 negatively regulates lin-14, which acts as a stage-specific inhibitor of dauer larva initiation. lin-4 and lin-14 mutations not only cause precocious or delayed dauer larva initiation globally but also affect individual cells to cause incomplete dauer larva morphogenesis. We interpret this latter defect as a result of partial activation of dauer larva initiation. lin-28 negatively regulates lin-29 to prevent lin-29 from interfering with a step in the differentiation of dauer larva hypodermis. Further analysis, including the use of genetic mosaics, is required to test various aspects of this model.

Our observation that eliminating both lin-28 and lin-29 gene products can restore complete dauer morphogenesis suggests that the wild-type lin-28 product allows complete dauer larva morphogenesis by preventing the inappropriate activity of lin-29 during early larval stages. In the absence of lin-29 gene product, lin-28 is not necessary for dauer larva morphogenesis. This situation involving lin-28 and lin-29 is analogous to one involving the nanos and hunchback genes of Drosophila (Hulskamp et al. 1989; Irish et. al. 1989; Struhl 1989). Mutations in the posterior group gene nanos, which result in embryos lacking all abdominal segments, can be suppressed by eliminating the maternal activity of the gap gene hunchback. Thus, nanos

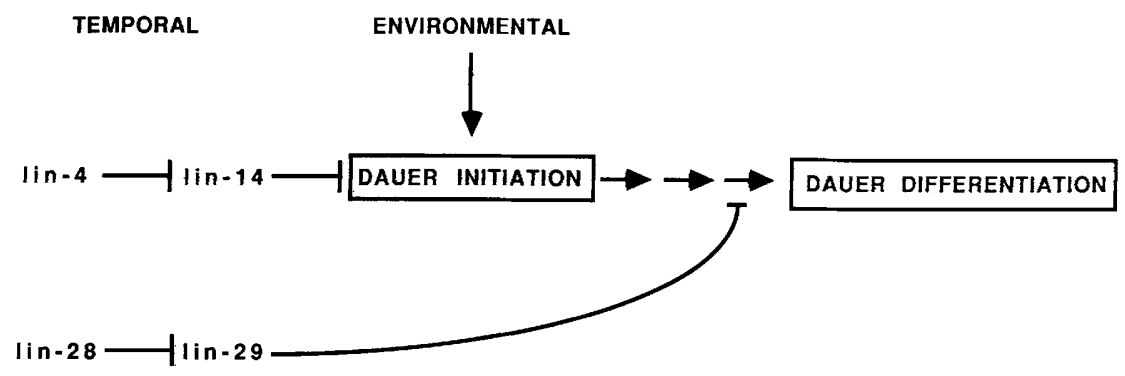

Figure 4. A model summarizing the temporal regulation of dauer larva initiation and dauer larva differentiation and showing the integration of temporal and environmental controls of dauer larva initiation. lin-4 negatively regulates lin-14, which regulates the stage specificity of dauer larva initiation. lin-28 functions to prevent lin-29 from interfering with a step in dauer larva differentiation. The environmental control may converge with the temporal control within specific cells by regulating a common regulator of dauer larva initiation. Arrows indicate positive regulatory interaction; bars, negative regulatory interaction. 
gene product normally functions to repress the maternal activity of hunchback in the posterior region of the egg and thus allow normal abdominal development. In the absence of maternal hunchback, nanos is not necessary. Thus, nanos and lin-28 represent a class of regulators that are not required to specify particular programs (in these cases, abdominal development and dauer larva morphogenesis, respectively) but, rather, block the activity of certain other genes that interfere with those programs.

\section{Integration of separate pathways for temporal and environmental control of dauer larva formation}

A genetic pathway for the reception and processing of environmental signals for dauer larva formation has been defined by epistasis analysis of dauer-constitutive and dauer-defective mutations (Riddle et al. 1981). Some of the daf genes in this pathway appear to be involved in the transduction or reception of environmental signals (Riddle et al. 1981; Perkins at al. 1986). Because none of the identified dauer-constitutive mutations alter the stage at which dauer larva initiation occurs, and lin-4 and lin-14 mutations do not bypass the environmental control, the pathway of environmental control defined by daf genes is separate from the pathway of temporal control defined by $l i n-4$ and $l i n-14$ genes. There are various plausible models for how these two separate pathways may converge to integrate temporal and environmental information and thus regulate a decision to initiate dauer larva development. Perhaps, a switch gene that controls the initiation of dauer larva development is regulated by both pathways (Fig. 4). Resolution of this issue awaits the genetic identification of additional genes affecting the timing of stage-specific dauer larva initiation and/or molecular identification of target genes regulated in common by daf genes and by lin-14.

\section{Methods}

\section{General methods and genetic markers}

General methods for the culture and handling of $C$. elegans have been described by Brenner (1974). Methods used for Nomarski differential interference contrast microscopy of living animals and for photography have been described by Sulston and Horvitz (1977) and Sternberg and Horvitz (1981).

The wild-type parent of all strains used in this work is $C$. elegans var. Bristol strain N2. The genetic markers listed below are from the Cambridge collection (Brenner 1974), unless otherwise noted. lin-4, lin-14, lin-28, and lin-29 alleles are described in Chalfie et al. (1981), Ambros and Horvitz (1984, 1987), and Ambros (1989), unless otherwise noted. The lin-14(n355n679ts) mutation was derived by reverting the semidominant lin-14(n355) mutation, thus containing both the original semidominant mutation $n 355$ and the new temperature-sensitive intragenic suppressor $n 679 t s$. lin-14(n355n679ts) at $15^{\circ} \mathrm{C}$ behaves as a semidominant, gain-of-function mutation that results in retarded defects in diverse cell types. At $25^{\circ} \mathrm{C}$, this mutation exhibits precocious defects in the same sets of lineages. Similarly, lin-14(n536n838) mutation contains both the original semidominant, gain-of-function lin-14(n536) mutation and the intragenic suppressor $n 838$. Therefore, the double mutation n536n838 is recessive and causes precocious defects in diverse cell lineages. This paper conforms to the standard C. elegans genetic nomenclature (Horvitz et al. 1979). Genetic markers used are LGI, lin-28(n719), lin-28(n947); LGII, lin-4(e912), lin-29(n333), lin-29(n1440) (a gamma ray-induced, recessive loss-of-function allele), dpy-10(e128), unc-52(e444); LGIII daf-7(e1372ts); LGV, him-5(e1490) (Hodgkin et al. 1979); and LGX, dpy-6(e14), lin-14(n536), lin-14(n355), lin-14-(ma135), lin-14(ma145), lin-14(n536n838), lin-14(n355n679ts), lin-14(n179ts), $\operatorname{lin}-14(n 727), \operatorname{lin}-14(n 360)$. The reciprocal translocation $\operatorname{szT}(X ; I)$ is a balancer for LGX and LGI that causes recessive lethality in hermaphrodites and carries wild-type alleles of lin-14 and lin-28 (Fodor and Deak 1982). mnC1 is a chromosomal rearrangement that suppresses crossing over in the LGII region (Herman 1978) and contains the recessive markers $d p y-10(e 128)$ and unc-52(e444) and wild-type alleles of lin-4 and lin-29. Strains used in this work are CB912 lin-4(e912), MT333 lin-29(n333), MT1149 lin-14(n536), MT1155 lin-4(e912)/mnC1; him-5(e1490), MT1388 lin-14(n355n679ts), MT1397 lin-14(n179ts), MT1524 lin-28(n719), MT1537 lin-28(n719);lin-14(n536), MT1538 lin-28(n719);lin-4 (e912), MT1836 lin-4(e912) lin-29(n333), MT1842 lin-14(n536n838), MT1848 lin-14(n360), MT1851 lin-14(n727), MT1852 lin-4(e912);ilin-14(n727), MT1999 lin-4(e912);lin-14 (n355n679ts), MT2015 lin-28(n947), VT116 lin-28(n719);1in-14 (n179ts), VT181 lin-14(n536) dpy-6(e14)/szT1(X;I), VT198 daf2(e1372ts);in-14(n727), VT291 lin-4(e912);lin-14(ma135), VT292 lin-14(ma135), VT334 lin-28(n719)/lin-28(n719) szT1(X;I);lin-14(ma135)/szT1(X;I), VT340 lin-14(ma145), VT387 lin-29(n1440)/mnC1; him-5(e1490), VT390 lin-29 (n1440);lin-14(n355n679ts), VT391 lin-29(n1440);lin-14 (ma135), VT392 lin-4(e912);1in-14(n179ts), VT393 lin-28 (n719);lin-29(n333), VT394 lin-28(n719);lin-29(n1440), and VT395 lin-29(n1440);lin-14(n536) dpy-6(e14).

\section{SDS selection of dauer larvae}

Dauer larvae were selected with SDS, as described by Cassada and Russell (1975). In general, worms from starved plates were washed off with sterile M9 solution, spun down in a tabletop centrifuge (1000 rpm, $3 \mathrm{~min}$ ), and resuspended in $1 \%$ SDS for 30 min at room temperature. After SDS treatment, worms were washed two to three times with sterile M9 and dropped on to culture plates seeded with Escherichia coli.

\section{Determining dauer-defective phenotype}

Mutants that consistently failed to give any SDS-resistant animals after repeated selection of starved cultures were considered dauer defective. We verified the dauer-defective phenotype of lin-4(e912) and lin-14(n536) mutants by introducing both mutations into a daf-7(e1372ts) (Swanson and Riddle 1981) background, a strong temperature-sensitive dauer-constitutive mutation. We failed to isolate any SDS-resistant dauer larvae, nor did we observe any animals exhibiting dauer larva morphology on plates cultured at the nonpermissive temperature $\left(25^{\circ} \mathrm{C}\right)$. We have also verified the dauer-defective phenotype of lin-29(n1440); lin-14(n536) dpy-6(e14) by culturing the strain as a heterozygote, lin-29(n1440);lin-14(n536) dpy-6(e14) ++ , and then checking the genotype of dauer larvae formed on starved plates. In no case did we observe dauer larva of the homozygous lin-29(n1440);lin-14(n536) dpy-6(e14) genotype.

\section{Scoring precocious and retarded dauer larvae}

After SDS selection, dauer larvae were examined using the dissecting microscope at $25-50 \times$. Precocious dauer larvae were 
identified by their unusually small size relative to wild-type dauer larvae, and their developmental stage was verified using high-magnification Nomarski differential interference contrast microscopy, as described below. Similarly, retarded dauer larvae were identified using the dissecting microscope after SDS selection by their unusually large size, and their developmental stage was verified using Nomarski optics. Dauer larvae of apparently normal size were also counted, and the developmental stage was confirmed using Nomarski optics. The penetrance of retarded or precocious dauer larva formation was derived by measuring the ratio of SDS-resistant dauer larvae formed at abnormal stages to the total number of SDS-resistant dauer larvae. We found that these results vary from culture to culture and probably depend on the population stage distribution at the time of starvation.

Developmental stages were determined using Nomarski optics of living dauer larvae to score for the occurrence of particular developmental events that are unperturbed in their timing in heterochronic mutants. The chief criteria used in this study were the number of gonadal nuclei and the extent of gonad morphogenesis (Kimble and Hirsh 1979). To verify that the stage-specific anatomical markers are reliable criteria for staging precocious dauer larva, a synchronized population of daf-7(e1372ts);lin-14(n727) Ll larvae were followed continuously from hatching to the time of dauer larva formation at the first (L1) molt. The synchronized population was obtained by treating gravid adult hermaphrodites with a solution of $1 \%$ sodium hypochlorite and $0.5 \mathrm{~N} \mathrm{NaOH}$ at room temperature for $7 \mathrm{~min}$ (Emmons et al. 1979). The surviving eggs were washed three times with sterile M9 and hatched in sterile M9 overnight. These arrested, undeveloped $\mathrm{L} 1$ larvae were fed and cultured at the nonpermissive temperature, $25^{\circ} \mathrm{C}$, and their subsequent development was followed by intermittent observation at 25-50 $\times$ magnification, using the dissecting microscope, and at $1000 \times$ using Nomarski optics.

\section{Scoring incomplete dauer larvae}

The SDS-resistant dauer larvae isolated from starved cultures were placed onto culture plates seeded with $E$. coli. Incomplete dauer larvae were identified, using the dissecting microscope at $25-50 \times$, by their unusual morphology (Fig. 2). Developmental stages and incompletely differentiated morphology were confirmed using Nomarski optics. The penetrance of incomplete dauer larva differentiation was calculated as the number of incomplete dauer larvae divided by the total number of SDS-resistant dauer larvae.

\section{Acknowledgments}

We are grateful to Lynn Carta, Sue Euling, Chip Ferguson, Iva Greenwald, Jan Kranz, Gary Ruvkun, Michael Shen, Paul Sternberg, Gary Struhl, Abby Telfer, and anonymous reviewers for their criticism, advice, and helpful discussion. This work was supported by U.S. Public Health Service research grant GM34028 and March of Dimes Birth Defects Foundation Basil O'Conner Starter research grant 5-445.

\section{References}

Albert, P.S., S.J. Brown, and D.L. Riddle. 1981. Sensory control of dauer larva formation in Caenorhabditis elegans. J. Comp. Neurol. 198: 435-451.

Albert, P.S. and D.L. Riddle. 1988. Mutants of Caenorhabditis elegans that form dauerlike larvae. Dev. Biol. 126: 270-293.
Ambros, V. and H.R. Horvitz. 1984. Heterochronic mutants of the nematode Caenorhabditis elegans. Science 226: 409416.

1987. The lin-14 locus of Caenorhabditis elegans controls the time of expression of specific post-embryonic developmental events. Genes Dev. 1: 398-414.

Ambros, V. 1989. A hierarchy of regulatory genes controls a larval-to-adult developmental switch in C. elegans. Cell 57: 49-57.

Baker, B.S. and K. Ridge. 1980. Sex and the single cell: On the action of major loci affecting sex determination in Drosophila melanogaster. Genetics 94: 383-423.

Brenner, S. 1974. The genetics of Caenorhabditis elegans. Genetics 77: 71-94.

Cassada, R.C. and R.L. Russell. 1975. The dauer larva, a postembryonic developmental variant of the nematode Caenorhabditis elegans. Dev. Biol. 46: 326-342.

Chalfie, M., H.R. Horvitz, and J.E. Sulston. 1981. Mutations that lead to reiterations in the cell lineages of C. elegans. Cell 24: 59-69.

Emmons, S.W., M.R. Klass, and D. Hirsh. 1979. Analysis of the constancy of DNA sequences during development and evolution of the nematode Caenorhabditis elegans. Proc. Nat1. Acad. Sci. 76: 1333-1337.

Evans, A.A.F. and R.M. Perry. 1976.Survival strategies in nematodes. In The organization of nematodes (ed. N.A. Croll), pp. 383-424. Academic Press, New York.

Fodor, A. and P. Deak. 1982. Isolation and phenocritical periodanalysis of conditional and non-conditional developmental mutants in C. elegans. Acta Biol. Acad. Sci. Hungaricae 32: 229-239.

Golden, J.W. and D.L. Riddle. 1984. The Caenorhabditis elegans dauer larva: Developmental effects of pheromone, food, and temperature. Dev. Biol. 102: 368-378.

Gould, S.J. 1977. Ontogeny and phylogeny. Harvard University Press, Cambridge.

Greenwald, I.S., P.W. Sternberg, and H.R. Horvitz. 1983. The lin-12 locus specifies cell fates in Caenorhabditis elegans. Cell 34: 435-444.

Herman, R.K. 1978. Crossover suppressors and balanced recessive lethals in C. elegans. Genetics 88: 49-65.

Hodgkin, J. 1980. More sex-determination mutants of C. elegans. Genetics 96: 649-664.

1985. Males, hermaphrodites and females: Sex determination in C. elegans. Trends Genet. 1: 85-88.

Hodgkin, J., H.R. Horvitz, and S. Brenner. 1979. Nondisjunction mutants of the nematode C. elegans. Genetics 91: 67-94.

Horvitz, H.R., S. Brenner, J. Hodgkin, and R.K. Herman. 1979. A uniform genetic nomenclature for the nematode Caenorhabditis elegans. Mol. Gen. Genet. 175: 129-133.

Hulskamp, M., C. Schroder, C. Pfeifle, H. Jackle, and D. Tautz. 1989. Posterior segmentation of the Drosophila embryo in the absence of a maternal posterior organizer gene. Nature 338: $629-632$.

Ingham, P.W. 1988. The molecular genetics of embryonic pattern formation in Drosophila. Nature 335: 25-34.

Irish, V., R. Lehmann, and M. Akam. 1989. The Drosophila posterior-group gene nanos functions by repressing hunchback activity. Nature 338: 646-648.

Kenyon, C. 1986. A gene involved in the development of the posterior body region of C. elegans. Cell 46: 477-487.

Kimble, J. and D. Hirsh. 1979. Post-embryonic cell lineages of the hermaphrodite and male gonads in Caenorhabditis elegans. Dev. Biol. 87: 286-300.

Lewis, E.B. 1978. A gene complex controlling segmentation in Drosophila. Nature 276: 565-570. 
Lewis, J.A. and J.A. Hodgkin. 1977. Specific neuroanatomical changes in chemosensory mutants of the nematode Caenorhabditis elegans. J. Comp. Neurol. 172: 485-510.

Miller, L.M., J.D. Plenefish, L.P. Casson, and B.J. Meyer. 1988. xol-1: A gene that controls the male modes of both sex determination and $\mathrm{X}$ chromosome dosage compensation in $\mathrm{C}$. elegans. Cell 55: 167-183.

Osche, G. 1963. Morphological, biological and ecological considerations in the phylogeny of parasitic nematodes. In The lower metazoa, Comparative biology and phylogeny (ed. E.C. Doughertyl, pp. 283-302. University of California Press, Berkeley.

Perkins, L.A., E.M. Hedgecock, J.N. Thompson, and J.G. Culotti. 1986. Mutant sensory cilia in the nematode Caenorhabditis elegans. Dev. Biol. 117: 456-487.

Riddle, D.L. 1977. A genetic pathway for dauer larva formation in Caenorhabditis elegans. Stadler Genet. Symp. 9: $101-$ 120.

Riddle, D.L., M.M. Swanson, and P.S. Alberts. 1981. Interacting genes in nematode dauer larva formation. Nature 290: 668671.

Riddle, D.L. 1988. The dauer larva. In The nematode Caenorhabditis elegans (ed. W.B. Wood and the community of C. elegans researchers), pp. 393-412. Cold Spring Harbor Laboratory, Cold Spring Harbor, New York.

Ruvkun, G. and J. Giusto. 1989. The Caenorhabditis elegans heterochronic gene lin-14 encodes a nuclear protein that forms a temporal developmental switch. Nature 338: 313319.

Singh, R.N. and J.E. Sulston. 1978. Some observations on molting in Caenorhabditis elegans. Nematologica 24: 6371.

Struhl, G. 1989. Differing strategies for organizing anterior and posterior body pattern in Drosophila embryos. Nature 338: $741-744$.

Sternberg, P.W. and H.R. Horvitz. 1981. Gonadal cell lineages of the nematode Panagrellus redivivus and implications for evolution by modification of cell lineage. Dev. Biol. 88: $147-166$.

Sulston, J.E. and H.R. Horvitz. 1977. Post-embryonic cell lineages of the nematode Caenorhabditis elegans. Dev. Biol. 56: $110-156$.

Swanson, M.M. and D.L. Riddle. 1981. Critical periods in the development of Caenorhabditis elegans dauer larva. Dev. Biol. 84: 27-40.

Taylor, A.L. and J.N. Sasser. 1978. Biology, identification and control of root-knot nematodes (Meloidogyne species). North Carolina State University Graphics, Raleigh. 


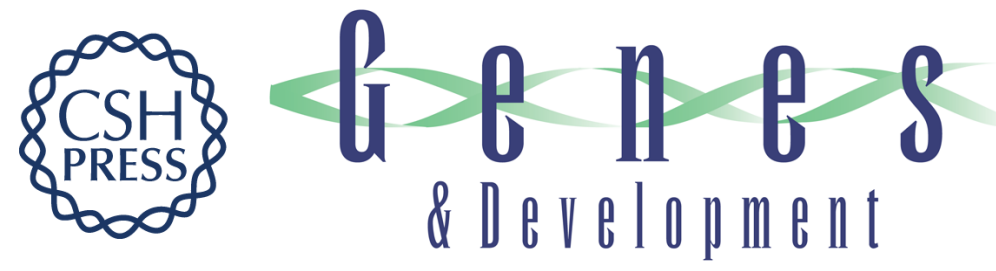

\section{Heterochronic genes control the stage-specific initiation and expression of the dauer larva developmental program in Caenorhabditis elegans.}

Z C Liu and V Ambros

Genes Dev. 1989, 3:

Access the most recent version at doi:10.1101/gad.3.12b.2039

References This article cites 35 articles, 8 of which can be accessed free at:

http://genesdev.cshlp.org/content/3/12b/2039.full.html\#ref-list-1

License

Email Alerting Service

Receive free email alerts when new articles cite this article - sign up in the box at the top right corner of the article or click here.

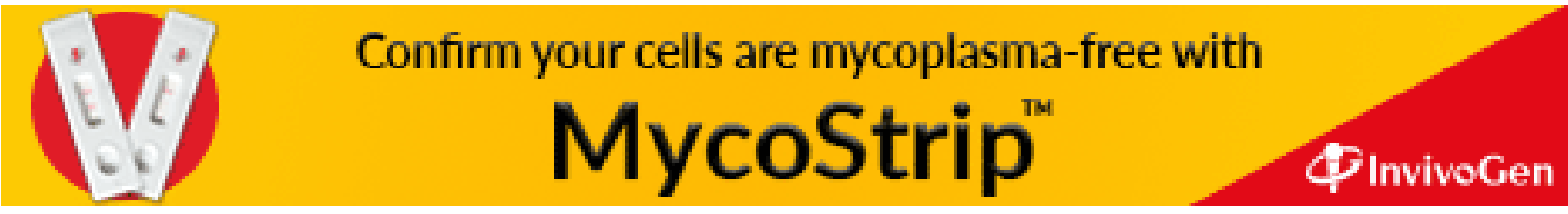

